\title{
The Evolving Demand for Negative Pressure Isolation Facilities during COVID 19: Trial of the Negative Pressure Isolation Tent
}

Fatimah Lateef FRCS (A\&E) MBBS FAMS (Em Med)1*, Sunny Chia S H2, Alan Teo H B3, Tan Teck Choon4

1Senior Consultant, Dept of Emergency Medicine, Singapore General Hospital, Professor, Duke NUS Graduate Medical School Professor, Yong Loo Lin School of Medicine, National University of Singapore. Director, SingHealth Duke NUS Institute of Medical Simulation (SIMS)

2Business Development Manager

3Director, Airtech Equipment Pvt Ltd

4Office for Service Transformation Singapore Health Services Pte Ltd

*Corresponding Author: Fatimah Lateef, Senior Consultant, Dept of Emergency Medicine, Singapore General Hospital, Professor, Duke NUS Graduate Medical School Professor, Yong Loo Lin School of Medicine, National University of Singapore. Director, SingHealth Duke NUS Institute of Medical Simulation (SIMS)

Received date: June 16, 2020; Accepted date: June 26, 2020; Published date: July 31, 2020

Citation: Lateef F, Sunny Chia S H, Alan Teo H B, Tan Teck C. (2020) The Evolving Demand for Negative Pressure Isolation Facilities during COVID 19: Trial of the Negative Pressure Isolation Tent. Biomedical Research and Clinical Reviews. 1(2); DOI: 10.31579/2692-9406/011

Copyright:@2020 Fatimah Lateef, This is an open-access article distributed under the terms of the Creative Commons Attribution License, which permits unrestricted use, distribution, and reproduction in any medium, provided the original author and source are credited.

\begin{abstract}
With COVID 19, more than ever before, healthcare institutions are realizing the need to develop, implement, assess, review and revise their infection control policies and guidelines of practice. In many facilities, patients who are known to be or suspected to be infectious are physically isolated from other patients and people. This is the rightful and ethical thing to do. These patients are usually identified through surveillance or using clinical and/ or microbiological criteria. Whilst practices such as personal and hand hygiene, safe infection control practices, distancing and others are useful, the use of formal isolation in hospitals represent an important step. This is also essential to reduce spread to healthcare workers.

In this paper, we describe a Negative Pressure Full Isolation Tent (NPFIT) which we tried out via simulation and the conduct of a simple smoke test to understand more on the concept of negative pressure and what it entails. It is our hope that readers will get a better understanding of this concept, through our simplified demonstration and trial.
\end{abstract}

Key words: isolation, covid-19, negative pressure, smoke test, negative pressure tent

\section{Introduction}

COVID 19 posed multiple challenges in every sector, including healthcare, which is leading the operations during the pandemic. Healthcare workers are not immune to the infection, even as they lead the way in managing high numbers of patients. They too have to observe the necessary distancing, contact tracing and infection control measures which are implemented. At work, they have to adhere strictly to PPE (personal protective equipment) guidelines in managing patients. In many healthcare institutions, new, modular team-based rosters have been implemented to reduce cross-interaction between staff. [1,2] In fact, departments are also planning strategies in the event that they are faced with a situation whereby the whole team may have to be quarantined due to one or more members being affected or testing COVID 19 positive. [3, 4]

The issue of isolation and air filtration is proving to be a critical consideration for the health and safety of healthcare workers. With each infectious disease outbreak, it is becoming more apparent on the need to address this, as part of the general infection control measures. [2, 5] Today, isolation facilities are equipped with negative pressure rooms and wards. This is fast becoming a standard requirement in hospitals and healthcare institutions. Even Emergency Departments (EDs), which represent the frontline of the hospitals, are equipped with negative pressure rooms and resuscitation cubicles today. This has become an essential requirement in view that the infectious patients presenting to the ED are often undifferentiated. [3, 5, 6]

\section{All about Negative Pressure}

A negative pressure room refers to a volumetric space whereby the internal atmospheric pressure is lower than the spaces into which it will open. This negative pressure within isolation rooms will help to control the airflow so that the number of airborne infectious particles and bioaerosols are reduced to a level which ensures cross-infection of other persons within the healthcare facility is unlikely. These isolation rooms must be very well-sealed in order to prevent excess air leakages into or out of the room. Preferably, the windows are those that do not open. The tighter the room is sealed, the better the differential pressure can be maintained.[2,5] The recommended pressure difference between these airborne infection isolation rooms and the corridor or the adjacent ambient pressure areas should be maintained at about $30 \mathrm{~Pa}$ if the room has an ante- 
room and at $15 \mathrm{~Pa}$, if the room does not have an ante-room. The air exchange rate recommended is at least 12 exchanges per hour. (5- 8)

In many general hospitals and healthcare facilities, only a certain percentage of their rooms are negative pressure rooms. As a result of this, many faced the need to come up with temporary negative pressure wards and rooms, at relatively short notice during this COVID 19 pandemic. Others found that they had to convert normal wards as well as even their standard positive pressure operating theatres into negative pressure environment, in order to accommodate the high numbers of patients during this pandemic. $[9,10]$ In general, these negative pressure isolation rooms are for patients who require droplet isolation, such as those with measles, varicella, tuberculosis, meningococcemia and of course, COVID 19.

Usually the numbers of isolation rooms required in an institution or healthcare facility is determined by several factors:

1. Epidemiological trends of the infectious disease in the local population

2. The local (and/ or regional) population catchment. It is also important to consider cross border travels and transfers, as well as

3. The healthcare institution offer of specialty services

Besides these, looking at some trends and statistics is also pertinent:

1. The numbers of patients admitted with infectious disease throughout the year, including during seasonal outbreaks in the country (eg. influenza season), with the projected numbers in the event of a pandemic. The seasonal variations in disease profile will help predict the load and requirement. Looking at trends over the last few years will also be helpful in planning a ball park figure in terms of numbers.

2. The average period of isolation required. This is dependent on the type of infectious disease we are referring to. It will give us an estimation of how long each isolation room will be occupied and thus the average occupancy rate

3. The need to address new and emerging infectious diseases must also be borne in mind.

\section{The Prerequisites for Isolation Rooms}

Isolation rooms require considerable resources to construct and maintain. The design goals must be clear from the conception phase. The idea is to be able to maintain an adequate level of infection protection in the environment surrounding the infectious patient. At the same time, it must be one of the prongs of the multiple strategies used to help reduce the threat to healthcare personnel entering the isolation rooms, as well as those outside the room. [2] Ventilation control is the key component of aerosol containment in the isolation rooms. Usually the ventilation engineer will work closely with the clinical team, Infectious Diseases physicians and the local infection control team in planning this. [6, 7]

The three factors which have to be monitored and looked into in this containment strategy are: $[5,7]$

1. Source control or removal

2. Dilutional rate: the higher the dilutional rate the more efficient the removal of the contaminated air. In fact, controlling the dilutional rate is said to be the single most important engineering control in the prevention of transmission. The dilutional time refers to the contaminant removal rate. [11,12]
3. The flow direction: it is important to ensure the outflow is safely exhausted and does not flow to other patient care areas. In the negative pressure rooms, the exhaust rate must exceed the supply rate by a generous margin. The Communicable Diseases Centre (CDC) in the USA recommends the exhaust rate from the isolation room exceeds the intake of air by about $10-15 \%$ or 50 cubic feet per minute. The direction of flow is said to be more important than the pressure differential through which the air flows. All air leaving the isolation rooms must be in a controlled fashion and is usually discharged to the exterior, away from windows and ventilation inlets.

Often overlooked is the presence of stagnant and underventilated areas, where the infectious aerosols and materials can accumulate and concentrate. [5, 12, 13] Thus, these "dead spaces" must be a point to discuss with the engineering team.

Some isolation rooms have an ante-room and toileting facilities attached. It is essential to maintain negative pressure in the isolation room with respect to the ante-room, when the door is opened. Similarly, the anteroom must maintain a negative pressure with respect to the surrounding or the corridor, when the door is opened. Ante-rooms help to maintain pressure gradients and reduce the migration of infectious particles into the corridors. [11, 12, 13] Ante-rooms also provide a space for the staff to don and doff their PPE or disinfect devices. In short, it represents a 'buffer zone'. The other important consideration is the air exchange rate (AER). This is a measure of how quickly contaminants can be removed from the area or zone. $[13,14]$ It is expressed as air exchange per hour $(\mathrm{AE} / \mathrm{Hr})$. The Total AER for an isolation room would then be guided by the formula:

Total AER $=$ Ventilation exhaust flow from the Isolation Room

\section{The Volume of the Isolation Room}

Air distribution that are employed should be designed to provide high effective ventilation rates. The other consideration for these negative pressure isolation rooms would be leakages. These can be planned leakages (eg. gaps under the doors, unsealed sides or top of the doors) or unplanned leakages (eg. through electrical plumbing outlets, floor-wall and wall-ceiling joints). [14, 15]

\section{The Need for Isolation Rooms}

All the factors discussed above are important considerations in planning a healthcare facility today. Singapore General Hospital, the largest teaching institution and a tertiary referral centre in Singapore, is part of the Academic Medical Centre with Duke-NUS Graduate Medical School. The hospital has 1700 beds, 36 different specialties, including NeuroSurgery and Cardiothoracic Departments as well as the only Burns Centre and ICU in the region. At any point in time, between $24 \%$ to $30 \%$ of these are isolation beds, whether single or cohort isolation, with negative pressure set up. During the COVID 19 pandemic, we also had 50 container isolation rooms set up at a large open carpark for our use. In addition to our usual Intensive Care Unit (ICU) beds, we were able to prepare and mobilize a total of 200 ICU bed spaces, including converting 40 operating theatres for potential ICU patient utilization.

Besides this we also explored other choices, as a standby potential in the event we experienced overwhelming numbers of Covid 19 patients needing isolation. One of these was the use of a Negative Pressure Full Isolation Tent (NPFIT). The NPFIT can be assembled in about 30 minutes. Being mobile and movable, gives the NPFIT the flexibility to be 
deployed where it is needed, at a suitably chosen location or space. We set the NPFIT up for testing in our simulation laboratory. (Figures 1,2)
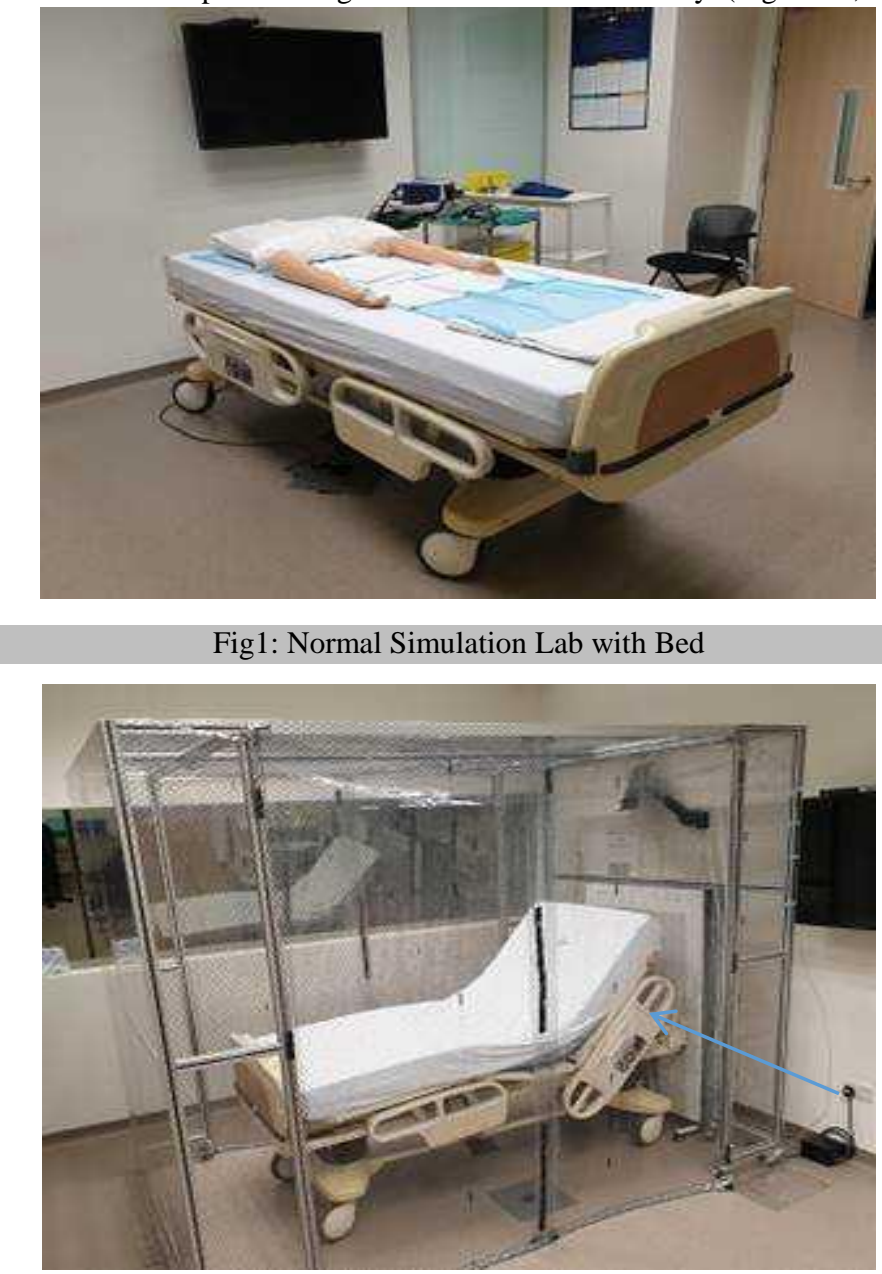

Fig 2: The Negative Pressure Full Isolation Tent over the Bed (the arrow represents the HEPA filter)
The frame is made from electro-galvanized steel and the "walls are transparent vinyl curtains. This tent has a length of $2.655 \mathrm{~m}$, width of $1.785 \mathrm{~m}$ and height of $2.115 \mathrm{~m}$. The volume of the tent is $10.02 \mathrm{~m} 3$. This is about a third of the size of a small patient room. There is a HEPA (High Efficiency Particulate Air) filter fitted at one end. The HEPA filter efficiency is set at $99.99 \%$ for particles $>0.3$ um size and will only need to be replaced once a year. The air velocity indicator is installed for monitoring and an air exhaust slit is present as well. [16-18]

To view the airflow direction within the tent, smoke was introduced via a smoke tube and the smoke visualization test was conducted to assess for leakages. (Figures 3, 4a, 4b) This was tested at different pressure levels; both over and under-pressure. When the HEPA filter is "ON", the smoke is seen to be drawn towards the filter and into it. (Fig 4a). When it is "Off", the smoke is seen to linger within the enclosure of the tent (Fig 3). Healthcare workers going into the tent must don their PPE and with the filter "ON". From the smoke test conducted, it would appear there will likely be a reduction of the aerosols suspended within the patient's space or domain.

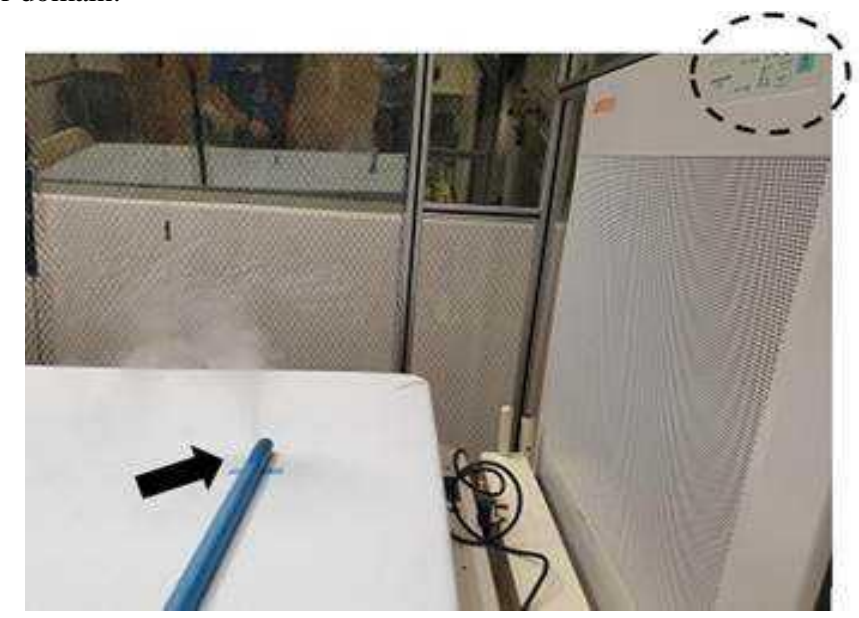

Fig 3: Introducing the "smoke": When the HEPA filter is in the OFF mode (circle) the smoke lingers around the enclosure of the tent pressure with the NPFIT

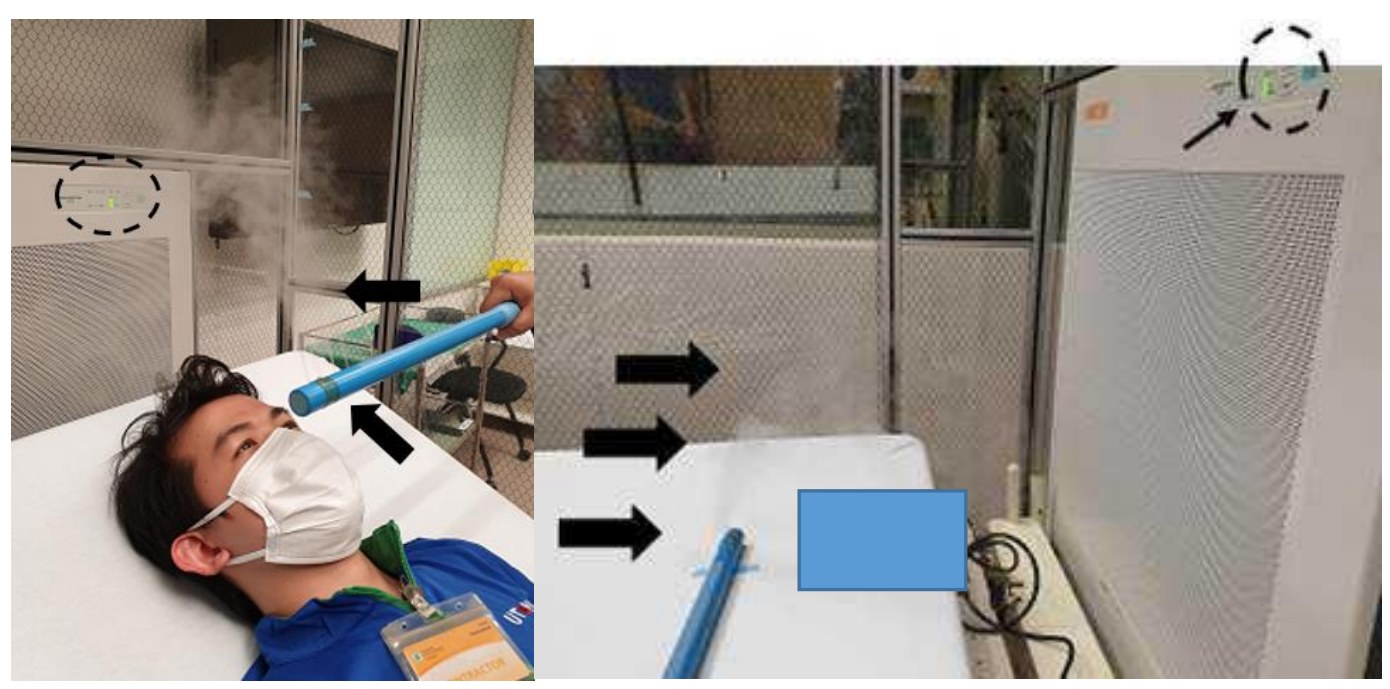

Fig 4a: When the HEPA filter is in the ON mode (circle) the smoke is now being drawn into the filter

Fig 4b: When the HEPA filter is in the ON mode and 'patient' is in Fowler's position, the smoke is being drawn into the filter 
Table 1 below shows comparison of air volume, air changes and pressure within the tent:

\begin{tabular}{|l|l|l|l|l|l|}
\hline & Powerful & High & Standard & Weak & Silent \\
\hline Air Velocity m/s & 0.46 & 0.26 & $\mathbf{0 . 2 0}$ & 0.16 & 0.12 \\
\hline Air Volume $\mathbf{~ m h}$ & 690 & 390 & $\mathbf{3 0 0}$ & 240 & 180 \\
\hline Air changes/hr & 68 & 38 & $\mathbf{2 9}$ & 23 & 17 \\
\hline Sound dBA & 58 & 45 & $\mathbf{4 3}$ & 40 & 36 \\
\hline
\end{tabular}

\section{Table 1: Comparison of air volume, air exchange and pressure with the NPFIT}

The recommendation is to maintain the parameters within the "Standard" range. (Table 1, in red). However there is the option to increase the air velocity and number of air exchanges per hour, especially for patients who are deemed to be more infectious, or the super-spreaders, if they have been identified. With the NPFIT set-up, it was noted that the higher the setting for the air velocity, the noise generated is also higher as measured in decibels (Table 1). The adjustments can be done appropriately as needed in the real patient care situation. In some cases, at night during sleeping time the air velocity may be lowered to ensure the 'noise' is more bearable to the patient.

In testing the time to achieve the negative pressure build-up, a digital micro-manometer was used to measure the pressure differential inside the tent (X) and the atmosphere (Y) outside the tent. Each test was conducted in the manner to determine the time required for the negative enclosure to achieve the highest negative pressure, using the different modes. (See Schematic Diagram below). Point X is at $1.32 \mathrm{~m}$ from the side of the tent and taken at 1 $\mathrm{m}$ above the ground. Point $\mathrm{Y}$ is $1 \mathrm{~m}$ away from the tent, also at $1 \mathrm{~m}$ above ground

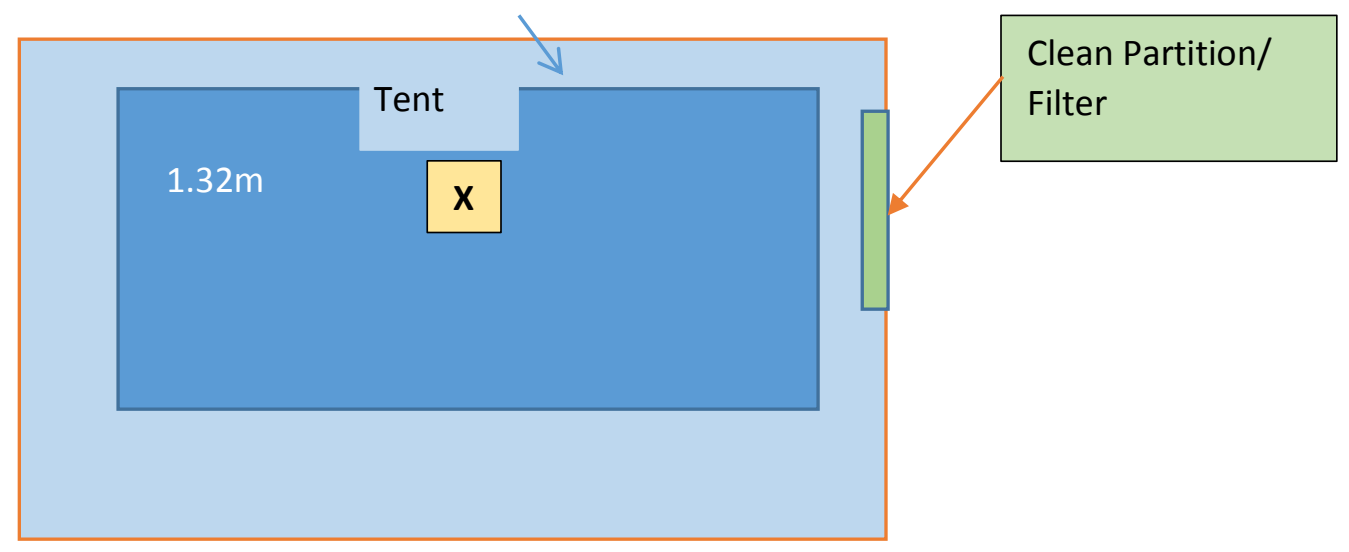

The time taken for the environment within the tent to achieve negative pressure at the different modes are reflected in Table 2 below. It can be seen that the time taken is relatively short, under 1 minute in general, for an enclosure the size of the NPFIT.

\begin{tabular}{|l|l|l|}
\hline Mode & Time in seconds & Pressure (Pa) \\
\hline Powerful & 45 & -11 \\
\hline High & 43 & -7.5 \\
\hline Standard & 42 & -6 \\
\hline Normal & 43 & -4 \\
\hline Quiet & 45 & -2.5 \\
\hline
\end{tabular}

\section{Table 2: Time taken to achieve negative pressure within the NPFIT}

Following the completion of use of the tent for any one infectious patient, terminal cleaning has to be carried out before it is used on the next infectious patient. This is the same type of cleaning conducted for hospitals rooms, cubicles and bays in which an infectious patient has been placed. Simply put, it represents an intense disinfecting procedure that involves removing every detachable item for disinfection. Light fixtures, air ducts and all surfaces from the ceiling to the floor must also be disinfected. All this is done using the usual hospital grade disinfectant. The NPFIT represents an option which can be utilized, in the event we require extra negative pressure rooms during an infectious disease outbreak. It is also an option for institutions which are not equipped with negative pressure isolation rooms.

\section{Conclusion}

As we continue to learn more about infectious diseases, and also with the new and emerging infectious diseases, the need for negative pressure isolation facilities will only increase. Healthcare facilities and institutions must continue to develop, implement, assess, review and revise infection control policies and measures based on patients' needs, infectious diseases characteristics and always bearing in mind, the threat of emerging new infectious agents and diseases. We must continue to 
develop innovation and capabilities in order to adequately prepare and be in control of a future outbreak or pandemic.

\section{References}

1. Lee JK, Jeong HW. (2020) Rapid expansion of temporary reliable airborne infection isolation rooms with negative air machines for critical Covid 19 patients. Am J of Infection Control 0:1-3.

2. Kao PH, Yang RJ. (2006) Virus diffusion in isolation room. Journal of Hospital Infection. 62:338-345

3. Lateef F. (2004) SARS changed the ED paradigm. Am J Emerg Med; 22: 483-487

4. Ministry of Health $(\mathrm{MOH})$. Attendances at Emergency Medicine Departments. Moh.gov.sg.2020.

5. Siegel JD, Rhinehart E, Jackson M, Chiarello 1. (2007) Healthcare Infection Control Practices Advisory Committee 2007 guidelines for isolation precautions: preventing transmission of infectious agents in healthcare settings. Am J Infect Control. 35(10 Suppl 2): S65-164

6. Miller SL, Clements N, Elliot SA, Subbash SS, Eagan A, Radonovich LJ. (2017) Implementing a negative pressure isolation ward for a surge in airborne infection patients. Am J of Infection Control 45: 652-659

7. Johnson DL, Lynch RA, Mead KR. (2009) Contaminant effectiveness of expedient patient isolation units. Am J of Infect Control; 37: 94-100

8. Winqvist C, Toomla S, Ahmed K, Kevnitski J, Mukkola R, Salomen $\mathrm{H}$. The effect of positive pressure in indoor air quality in a school building: a case study. Emergy Procedia 132: 165170
9. Mousain ES, Grosskopf KR. (2016) Airflow patterns due to door motion and pressurization in hospital isolation room. Sci Technol Built Environ; 22: 379-384

10. Chow TT, Kwan A, Lia Z, Bai W. (2006) Conversion of operating theatre from positive pressure to negative pressure environment. Journal of Hospital Infection 64: 371-378

11. Tang JW, Eames J. (2005) Door opening motion can potentially lead to transient breakdown in negative pressure isolation conditions: the importance of vorticity and buoyancy airflows. J of Hospital Infection; 61:283-286

12. Adams NJ, Johnson DL, Lynch RA. (2011) The effect of pressure differential and care provider movement on airborne infection isolation room containment effectiveness. Am $\mathbf{J}$ of Infect Control; 39:91-97

13. Mousavi ES, Grosskopf KR. (2016) Airflow patterns due to door motion and pressurization in hospital isolation rooms. Sci Technol Built Environ 22:379-394

14. Saravia SA, Raynor PC, Streifel AJ. (2007) A performance assessment of airborne infection isolation room. Am $\mathrm{J}$ of Infection Control 35:324-331

15. Walker JT, Hoffman P, Bennet AM, Vos MC, Thomas M, Tomlinson N. (2007) Hospital and community acquired infection and the built environment design and testing of infection control rooms. Journal of Hosp Infection 65:43-49

16. Operations Manual. Negative Pressure Isolation Tent. Airtech Equipment Pte Ltd.

17. Palmer B, Clark L. natural air filter Association Guidelines, 2016.

18. Heimbuch BK, Wu CY, Wander JD. Viral penetration of High Efficiency Particulate Air (HEPA) Filter. 